\title{
Autophagy-Dependent Beneficial Effects of Exercise
}

\author{
Jens Frey Halling and Henriette Pilegaard \\ Department of Biology, University of Copenhagen, 2100 Copenhagen, Denmark \\ Correspondence: hpilegaard@bio.ku.dk
}

Exercise has long been recognized as a powerful physiological stimulus for a wide variety of metabolic adaptations with implications for health and performance. The metabolic effects of exercise occur during and after each exercise bout and manifest as cumulative adaptive responses to successive exercise bouts. Studies on the beneficial effects of exercise have traditionally focused on the biosynthesis of metabolic proteins and organelles. However, the recycling of cellular components by autophagy has recently emerged as an important process involved in the adaptive responses to exercise. This review covers the regulation of autophagy by exercise, with emphasis on the potential autophagy-dependent beneficial effects of exercise.

\section{ADAPTATIONS TO EXERCISE TRAINING}

Fxercise training can induce multiple adaptaEtions in skeletal muscle, the type of which depends on the mode of exercise. Hence, resistance exercise training is characterized by increased muscle fiber cross-sectional area (hypertrophy), whereas endurance exercise training is characterized by metabolic adaptations in skeletal muscle.

Resistance exercise training-induced increases in muscle mass contribute to increased muscle strength and muscle performance (Chesley et al. 1992; Biolo et al. 1995) with importance for both athletic ability and everyday physical function. This is, in part, facilitated by a net increase in myofilament proteins. In accordance, a single resistance exercise bout has been shown to increase protein synthesis (Chesley et al.
1992; Biolo et al. 1995; Phillips et al. 1997) for up to 48 hours involving increased translational initiation. Of notice is, however, that resistance exercise also increases protein degradation for up to 24 hours (Biolo et al. 1995; Phillips et al. 1997) underlining the importance of a well-regulated balance between protein synthesis and degradation in the regulation of muscle mass.

Endurance exercise training-induced metabolic adaptations in skeletal muscle contribute to increasing the maximal oxidative capacity as well as the metabolic efficiency of skeletal muscle with concomitant improvements in endurance performance and health. Endurance exercise training-induced increases in content and activity of proteins involved in oxidative metabolism in human and rodent skeletal muscle are well established (Holloszy 1967; Gollnick et al. 1972; Henriksson and Reitman 1977). These

Editors: Juleen R. Zierath, Michael J. Joyner, and John A. Hawley

Additional Perspectives on The Biology of Exercise available at www.perspectivesinmedicine.org

Copyright (C) 2017 Cold Spring Harbor Laboratory Press; all rights reserved; doi: 10.1101/cshperspect.a029777

Cite this article as Cold Spring Harb Perspect Med 2017;7:a029777 
adaptations have, in general, been reported as a result of increased synthesis of metabolic proteins and investigations of the underlying mechanisms behind metabolic adaptations with exercise training have primarily focused on the regulation of protein synthesis. However, the original study by Henriksson and Reitman showing that the activity of oxidative enzymes in human skeletal muscle increased over the course of several weeks of endurance exercise training also showed that the gained enzyme activity was rapidly lost within $1-2$ weeks of detraining (Henriksson and Reitman 1977). This underlines the high turnover of metabolic proteins in skeletal muscle. Furthermore, more recent evidence suggests that the regulation of protein removal by autophagy is of equal importance as increased synthesis of metabolic proteins for obtaining the full beneficial metabolic effects of endurance exercise training.

\section{MECHANISMS OF AUTOPHAGY}

The term autophagy is derived from the ancient Greek words "auto" (self) and "phagein" (to eat). Autophagy is a conserved mechanism in which targeted cellular components are engulfed in maturing autophagosomes that subsequently fuse with lysosomes for hydrolytic degradation and recycling of proteins, membranes, and other cellular components.

Important knowledge has been gathered regarding key molecular mechanisms involved in initiation and execution of cellular recycling through autophagy, which have recently been comprehensively reviewed (Ktistakis and Tooze 2016). In brief, a central set of autophagy-related (ATG) proteins controlling autophagosome formation and elongation has been identified in yeast, most of which have been found to have one or more mammalian homologs. An essential step in autophagosome maturation is the recruitment of the ULK1 kinase complex (including ATG13, ATG101, and FIP200) and the class III PI 3-kinase (PI3K) complex (including VPS34, Beclin-1, ATG14, and p150) to the maturing autophagosome membrane (Fig. 1). The interaction between the ULK1 complex, LC3/GABARAP, and ATG5-12-16L1 at the au- tophagosome membrane increases the activity of ULK1 and sustains PI3K complex activity, which drives phosphatidylinositol 3-phosphate (PI3P) formation and promotes autophagosome maturation. Furthermore, ATG5-12-16L1-dependent phosphatidylethanolamine (PE) lipidation of LC3-I to LC3-II on the autophagosome membrane is crucial for autophagosome maturation (Fig. 1) (Mizushima and Komatsu 2011; Ktistakis and Tooze 2016). LC3-II and the LC3-II/LC3-I ratio are therefore widely used as markers for autophagosome content and autophagy flux, although interpretations of LC3-II as a general autophagy marker should be made cautiously (Mizushima and Yoshimori 2007; Rubinsztein et al. 2009; Klionsky et al. 2016). Although elevated LC3-II content can be interpreted as a marker of increased autophagosome content and therefore induction of autophagy, a reduction in LC3-II can, conversely, also be the result of increased autophagic flux, because LC3-II itself is degraded in the lysosome. Tracking of other proteins known to be degraded during autophagy can therefore be useful in interpreting alterations in autophagy (Klionsky et al. 2016).

\section{Selective Autophagy}

Although autophagy can function in a nonselective manner, for example to provide substrates during energy deprivation, mechanisms for selective clearance of specific organelles and proteins have been identified. The autophagy adaptor protein p62 contains a carboxy-terminal ubiquitin-associated domain and directly interacts with LC3-II, thereby facilitating the incorporation of ubiquitinated substrates into autophagosomes (Fig. 1). Because p62 is degraded during autophagy, changes in p62 protein content have together with LC3 lipidation been used as a surrogate marker for autophagy flux (Klionsky et al. 2016).

An emerging autophagy-dependent mechanism with potential implications for exercise training adaptations is the autophagic clearance of damaged mitochondria termed mitophagy and a molecular machinery that serves to target mitochondria for degradation has been discov- 
Autophagy-Dependent Beneficial Effects of Exercise

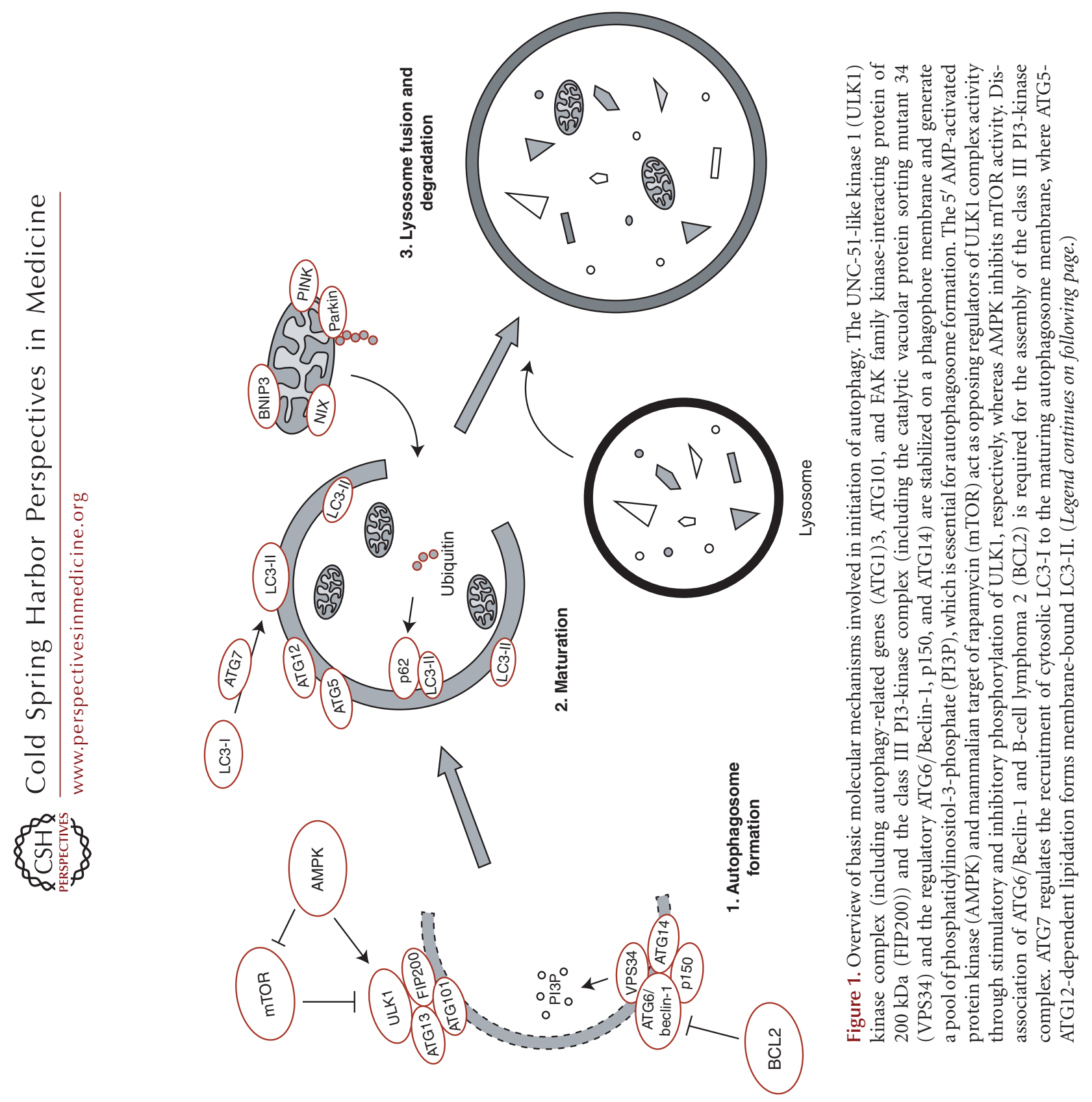


ered. The kinase PTEN-induced putative kinase 1 (PINK1) is stabilized on depolarized mitochondrial membranes, for example, in response to oxidative stress, and recruits the E3 ubiquitin ligase parkin to mitochondria. On activation, parkin ubiquitinates several mitochondrial membrane proteins, thereby targeting mitochondria for degradation. Furthermore, specific mitophagy receptors such as BCL2/adenovirus E1B $19-\mathrm{kDa}$ protein-interacting protein 3 (BNIP3) and NIP3-like protein X (NIX) present on mitochondrial membranes can directly interact with LC3-II to induce mitophagy (Fig. 1) (Youle and Narendra 2011).

\section{Acute Exercise-Induced Autophagy}

The ability of a single endurance exercise bout to induce an autophagic response in skeletal muscle was first described in 1984. Thus, using electron microscopy (EM), the formation of autophagic vacuoles was detected in mouse muscle fibers 2 to 7 days after an extremely strenuous $(9 \mathrm{~h}$ ) treadmill running bout (Salminen and Vihko 1984). However, the notion that exercise may stimulate autophagic removal of cellular components has only recently received widespread attention following reports that LC3-II as well as autophagosome content detected by EM was increased in mouse muscle after a single bout of treadmill running (Grumati et al. 2011). This finding has since been supported by several other studies in rodents (He et al. 2012; Jamart et al. 2012b; Pagano et al. 2014; Saleem et al. 2014; Fritzen et al. 2015; Liu et al. 2015; Vainshtein et al. 2015b; Halling et al. 2016). However, the autophagic response in human muscle to acute endurance exercise seems to be more complex. Hence, ultra-endurance running has been shown to potently increase LC3-II content (Jamart et al. 2012b), whereas exercise bouts of lower duration have been reported to lower the content of LC3-II (Fritzen et al. 2015; Moller et al. 2015; Schwalm et al. 2015). Moreover, some studies have observed unchanged skeletal muscle LC3II content in humans following a single cycling exercise bout (Masschelein et al. 2014; Tachtsis et al. 2016). In addition, reduced p62 content following endurance exercise has been reported in both mouse (He et al. 2012; Pagano et al. 2014) and human (Schwalm et al. 2015) skeletal muscle, although others have observed unchanged p62 content after a single endurance exercise bout (Jamart et al. 2012b; Fritzen et al. 2015; Moller et al. 2015; Halling et al. 2016; Tachtsis et al. 2016). Altogether, there is robust evidence suggesting that autophagy is stimulated in skeletal muscle in response to acute endurance exercise. However, the exact mechanism behind induction of autophagy may depend on factors such as intensity/duration of exercise (Schwalm et al. 2015), feeding (Jamart et al. 2013), and timing of muscle sampling (Halling et al. 2016), which may contribute to the variability of the observed autophagic responses to acute endurance exercise.

Several studies have also examined the effects of strength/resistance exercise on regulation of autophagy. It has been shown that LC3II protein and GABARAP mRNA were reduced from 3 to 24 hours after a single session of resistance exercise in both young and aged human muscle, which was interpreted as decreased activation of autophagy (Fry et al. 2013). Furthermore, it has been shown that the decreased LC-II content following resistance exercise was dependent on post-exercise intake of essential amino acids (Glynn et al. 2010), suggesting that resistance exercise-induced regulation of au-

Figure 1. (Continued) The autophagy adaptor protein p62 links ubiquitinated substrates with LC3-II. Selective autophagic clearance of mitochondria (mitophagy) is regulated by the kinase PTEN-induced putative kinase 1 (PINK1), which is stabilized on depolarized mitochondrial membranes and recruits the E3 ubiquitin ligase parkin to mitochondria. Parkin regulates ubiquitination of mitochondrial membrane proteins, thereby targeting mitochondria for degradation. The mitophagy receptors BCL2/adenovirus E1B 19-kDa protein-interacting protein 3 (BNIP3) and NIP3-like protein X (NIX) present on mitochondrial membranes can directly interact with LC3-II to induce mitophagy. Finally, the mature autophagosomes fuse with lysosomes causing hydrolytic degradation of autophagosomal cargo. 
Autophagy-Dependent Beneficial Effects of Exercise

tophagy is dependent on nutrient availability. However, the apparent modulation of autophagy by resistance exercise in combination with amino acid intake was not associated with changes in either overall protein synthesis or protein breakdown at 2 hours post-exercise (Glynn et al. 2010). This indicates that autophagy-mediated release of amino acids is not involved in anabolic/catabolic processes in the early recovery period. The reported lowering of LC3-II and LC3-II/I content in human skeletal muscle during the early recovery period after a resistance exercise bout has been recapitulated in several recent studies (Dickinson et al. 2016; Francaux et al. 2016; Smiles et al. 2016). This may suggest a deactivation of autophagy during recovery from resistance exercise, assuming that LC3-II/I is a valid marker for autophagic flux. In contrast, others have reported no change (Smiles et al. 2015) or increase (Ogborn et al. 2015) in autophagy markers at various timepoints after resistance exercise. However, as previously described, LC3-II itself is degraded when autophagosomes fuse with lysosomes suggesting that, in certain cases, a reduction in LC3-II content can actually be the result of increased autophagic flux (Mizushima and Yoshimori 2007; Rubinsztein et al. 2009). It is, therefore, noteworthy that a recent study showed resistance exercise-induced activation of chaperone-assisted selective autophagy and LC3 puncta formation in human skeletal muscle up to 24 hours into recovery (Ulbricht et al. 2015), similar to that observed after treadmill running in mice (Grumati et al. 2011; He et al. 2012). Therefore, it is uncertain how resistance exercise affects autophagic flux. An approach that may be used to address this is colchicineinduced blockade of lysosomal degradation as previously used to determine autophagic flux in mice in response to endurance exercise (Vainshtein et al. 2015b). However, rodent models of resistance exercise may not be comparable with humans, underlined by the observation that "resistance exercise" did not affect LC3-II levels in rat skeletal muscle (Ogasawara et al. 2016). On the other hand, the activity of VPS34, a component of the PI3K complex, has been shown to be increased following high-resistance electrically stimulated contraction of rat skeletal muscle (MacKenzie et al. 2009). This suggests that autophagy is activated during recovery from resistance exercise similarly to endurance exercise, although further studies are required to directly assess the effects of resistance exercise on autophagic flux in human muscle.

\section{MOLECULAR MEDIATORS OF EXERCISE- INDUCED AUTOPHAGY}

\section{AMPK}

Cell culture studies have indicated that the $5^{\prime}$ AMP-activated protein kinase (AMPK) regulates autophagy through ULK1 Ser-317 and Ser-555 phosphorylation (Meley et al. 2006; Egan et al. 2011) during conditions of cellular energy demand. Accordingly, ULK1 Ser-317, and Ser-555 phosphorylation has been shown to increase in mouse muscle in response to a single exercise bout (Pagano et al. 2014). Furthermore, it has been shown that the catalytic AMPK $\alpha 2$ subunit is required for exercise-induced LC3 lipidation and ULK1 Ser-777 phosphorylation in mouse skeletal muscle (Liu et al. 2015). The link between AMPK and autophagy is also supported by the observation that BCL2 AAA mutated (Thr69Ala, Ser70Ala, and Ser84Ala) mice with impaired dissociation of the Beclin-1/BCL2 complex, causing inhibition of PI3K complex formation and blunted exerciseinduced activation of autophagy, showed lower AMPK phosphorylation levels (He et al. 2012). On the other hand, inducible muscle-specific knockout $(\mathrm{KO})$ of ATG7 resulted in impaired exercise-induced autophagy, but did not affect AMPK phosphorylation in mouse skeletal muscle (Lo et al. 2014). Moreover, an association between exercise-induced AMPK activation, ULK1 phosphorylation and modulation of autophagy is supported by results from some human studies (Moller et al. 2015; Schwalm et al. 2015). However, others have reported that there was no correlation between exerciseinduced AMPK activation and LC3 lipidation in human muscle as well as no apparent effects on LC3 lipidation with pharmacological activation of AMPK in incubated mouse skeletal muscle or 
human myotubes (Fritzen et al. 2015). Still, AMPK seems to be persistently required for ULK1 Ser-555 phosphorylation (Egan et al. 2011; Fritzen et al. 2015). Taken together, evidence suggests that AMPK-dependent activation of autophagy can occur in skeletal muscle in response to acute exercise. Because AMPK is known as an intracellular energy sensor, this may suggest that exercise-induced autophagy activation occurs in response to lowered intracellular energy charge. However, a recent study showed that exercise-induced activation of autophagy was independent of continuous systemic glucose infusion during exercise (Moller et al. 2015), suggesting that activation of autophagy in skeletal muscle during exercise is not caused by insufficient substrate supply.

\section{mTOR}

The mammalian target of rapamycin (mTOR) has been identified as a major negative regulator of autophagy through an AMPK-opposing mechanism (Kim et al. 2011). During conditions of high substrate availability, a signaling pathway is activated involving the insulin-stimulated class I PI3K, which recruits Akt leading to activation of mTOR. Consequently, ULK1 is phosphorylated by mTOR at Ser-757, which, contrary to AMPK-mediated phosphorylation, inhibits formation of the ULK1 kinase complex preventing autophagosome maturation (Kim et al. 2011). AMPK activation inhibits mTOR signaling and, indeed, ULK1 Ser-757 phosphorylation has been shown to decrease in mouse skeletal muscle both during exercise (Pagano et al. 2014) and 10 hours into recovery (Halling et al. 2016) from a single treadmill running bout. This suggests that deactivation of mTOR signaling contributes to endurance exerciseinduced autophagy. In addition, autophagy activation following high-resistance electrically stimulated contraction of rat skeletal muscle correlated with mTOR activity, while leucine treatment, but not amino acid starvation, of C2C12 cells also increased VPS34 activity (MacKenzie et al. 2009). Furthermore, inhibition of mTOR signaling attenuated protein synthesis rates up to 24 hours after a single resistance exercise bout in rat skeletal muscle (Ogasawara et al. 2016). Together, this suggests that it may be the increased amino acid turnover per se that regulates autophagy through mTOR signaling in response to resistance exercise potentially allowing further stimulation of protein synthesis at later timepoints.

\section{FOXO}

Several stress-induced transcription factors have been proposed to regulate the expression of genes involved in autophagy (Pietrocola et al. 2013) of which the best-described example is the forkhead box O (FOXO) family of transcription factors. In particular, FOXO3 has been shown to play an important role in regulating core ATGs, including LC3 and BNIP3 in skeletal muscle (Mammucari et al. 2007; Zhao and Klionsky 2011). FOXOs are responsive to nutrient deprivation and oxidative stress through posttranslational modifications. This includes Akt (PKB)-mediated phosphorylation of FOXO3 on multiple residues leading to inhibition and retention of FOXO3 in the cytosol (Brunet et al. 1999). The observation that Akt 1 and $2 \mathrm{KO}$ in mice increased BNIP3 mRNA in skeletal muscle (Reynolds et al. 2012) supports the relevance of Akt-mediated FOXO3 regulation in autophagy. Furthermore, the findings that the AMPK activator, AICAR, increased FOXO3 phosphorylation with concomitant transient relocalization of $\mathrm{FOXO} 3$ to the nuclei and increase in mRNA content of autophagyrelated proteins in muscle cells (Sanchez et al. 2012) provide strong evidence that AMPK is involved in posttranslational regulation of FOXO3. In addition, acetylation of FOXO3 has been reported to induce translocation of FOXO3 to the cytosol followed by ubiquitination and degradation of $\mathrm{FOXO} 3$ in the proteasome, whereas sirtuin-mediated deacetylation and activation of FOXO3 has been suggested to promote autophagy (Bertaggia et al. 2012). Moreover, AMPK-induced modulation of sirtuin activity is thought to reduce FOXO3 acetylation. Taken together, multiple posttranslational modifications seem to contribute in the 
Autophagy-Dependent Beneficial Effects of Exercise

regulation of FOXO3 activity and localization. Interestingly, increased FOXO3 mRNA and protein content as well as stimulatory dephosphorylation of FOXO3 has been observed in human and mouse skeletal muscle following acute endurance exercise (Louis et al. 2007; Jamart et al. 2012a, 2013; Wang et al. 2015). This indicates that $\mathrm{FOXO} 3$ regulation, possibly mediated by Akt/AMPK, contributes to transcriptional regulation of autophagy components in response to exercise.

\section{TFEB}

The transcription factor EB (TFEB) has been shown to be a key factor in autophagy and lysosome function through transcriptional regulation of genes encoding autophagy and lysosome proteins (Settembre et al. 2011). TFEB localization and activity is regulated by mTORC1-mediated phosphorylation of multiple residues and dephosphorylation by calcineurin. In accordance, exhaustive running exercise in mice has been reported to induce a calcineurin-mediated dephosphorylation of TFEB in skeletal muscle with concomitant nuclear translocation of TFEB and overexpression of a calcineurin inhibitor in muscle by electroporation prevented these effects (Medina et al. 2015). A simultaneous exercise-induced reduction in mTOR-mediated phosphorylation and inhibition of TFEB has been suggested to contribute to ensuring that TFEB translocates to the nucleus in response to muscle contractions (Medina et al. 2015). Together, this shows the potential of TFEB to regulate exercise training induced long-term adaptations in the autophagy and lysosome pathways.

\section{p53}

The tumor suppressor p53 has also been shown to be involved in regulation of autophagy. However, whether p53 functions as an activator or suppressor of autophagy seems to be dependent on the context and subcellular localization, because cytosolic p53 has been shown to inhibit autophagy (Tasdemir et al. 2008), whereas p53 induces transcription of key stimulatory autophagy genes in the nucleus (Maiuri et al. 2009; Kenzelmann et al. 2013). Furthermore, the findings that the nuclear abundance of p53 is increased in human muscle (Tachtsis et al. 2016) and decreased in mouse muscle (Saleem and Hood 2013) during recovery from exercise underline the complexity of p53 regulation. Together, the possible role of p53 in the regulation of exercise-induced autophagy remains to be fully elucidated. However, the finding that p53 is dispensable for metabolic adaptations to exercise training in mice (Saleem et al. 2009) suggests that p53-mediated exercise-induced autophagy may not be required for the beneficial metabolic effects of exercise.

\section{PGC- $1 \alpha$}

The exercise responsive transcriptional co-activator peroxisome proliferator-activated receptor $\gamma$ coactivator (PGC) $-1 \alpha$ has been identified as a key regulator of mitochondrial biogenesis (Wu et al. 1999; Pilegaard et al. 2003; Lin et al. 2005). Moreover, several recent studies have suggested that PGC- $1 \alpha$ plays a role in exerciseinduced autophagy. Hence, the finding that muscle-specific PGC-1 $\alpha$ overexpression (MCK) was associated with increased BNIP3 protein content and increased basal autophagy (Lira et al. 2013) indicates that PGC- $1 \alpha$ contributes to the regulation of autophagy in resting skeletal muscle. This was accompanied by results showing that autophagy levels are higher in oxidative than glycolytic muscles (Lira et al. 2013). The finding that exercise increased LC3-II protein content during recovery from a single treadmill running bout in PGC-1 $\alpha$ MCK mice, but not in wild-type (WT) mice, suggests that elevated levels of PGC- $1 \alpha$ also contributes in inducing autophagy in response to acute exercise. This was supported by a blunted treadmill exerciseinduced increase in skeletal muscle LC3-II protein in muscle-specific PGC- $1 \alpha \mathrm{KO}$ mice (Halling et al. 2016). In addition, an attenuated treadmill exercise-induced increase in mitochondrial LC3-II flux in skeletal muscle from whole-body PGC- $1 \alpha \mathrm{KO}$ mice determined by monitoring LC3-II accumulation after treatment with the autophagy inhibitor colchicine 
further underlines the impact of PGC- $1 \alpha$ in exercise-induced autophagy (Vainshtein et al. 2015b). Together, this may reflect that the metabolic profile of PGC- $1 \alpha$ overexpression and $\mathrm{KO}$ mice affects the exercise-induced autophagy response. Thus, PGC-1 $\alpha$ may influence autophagy indirectly through regulation of muscle oxidative capacity. However, it is also possible that PGC- $1 \alpha$ affects exercise-induced autophagy through transcriptional regulation of autophagy proteins. Hence, the observations that PGC- $1 \alpha \mathrm{KO}$ mice had lower TFEB protein in skeletal muscle than WT mice (Vainshtein et al. 2015a) and did not increase the mRNA content of LC3, p62, and Niemann-Pick C1 in response to treadmill running (Vainshtein et al. 2015b) indicate that PGC- $1 \alpha$ also plays a role in the endurance-exercise-induced adaptive gene responses of autophagy proteins in skeletal muscle. Taken together, this suggests that PGC- $1 \alpha$ influences exercise training-mediated adaptations in key autophagy markers resulting in increased capacity of the autophagy machinery with exercise training.

\section{AUTOPHAGY-DEPENDENT BENEFICIAL EFFECTS OF EXERCISE}

\section{Exercise Performance and Oxidative Capacity}

The possibility that exercise-induced autophagy influences exercise performance is supported by the finding that BCL2 AAA mutated mice that are incapable of exercise-induced BCL2Beclin-1 disassociation and autophagy activation have lower maximal exercise capacity than control mice (He et al. 2012). Of notice is that the lower maximal exercise capacity was not because of differences in basal muscle properties, but the inability to induce autophagy during running. Maximal running distance was also shown to be lower in ATG6 (Beclin-1) ${ }^{+/-}$ mice with reduced ATG6 protein content in skeletal muscle, but normal basal autophagy (He et al. 2012), although others did not observe changes in time to exhaustion during treadmill running in ATG6 ${ }^{+/-}$mice (Lira et al. 2013). This suggests that the impact of the level of ATG6 on exercise performance may depend on exercise type, intensity, and/or duration. In accordance, exercise performance during regular treadmill running was not impaired in inducible skeletal-muscle-specific ATG7 KO mice, despite a blunted exercise-induced autophagy response (Lo et al. 2014), supporting that acute autophagy activation is not universally required for sustaining muscle contractions. However, the inducible deletion of ATG7 in skeletal muscle reduced performance and caused profound mitochondrial membrane depolarization in skeletal muscle during downhill running (eccentric muscle contractions) in female mice (Lo et al. 2014), suggesting that autophagy influences exercise performance during more damaging muscle contractions (Fig. 2).

Several studies suggest that autophagy is essential for cellular adaptations to exercise training. A potential link between exercise-induced autophagy and exercise training-mediated adaptations was examined using ATG6 ${ }^{+/-}$mice. Exercise training did not improve endurance exercise performance and did not increase the protein content of the oxidative markers cytochrome $c$ and COXIV, or LC3 and BNIP3 protein in skeletal muscle of $\mathrm{ATG}^{+/-}$mice (Lira et al. 2013). This suggests that exerciseinduced autophagy is required for exercise training-mediated adaptations in skeletal muscle oxidative capacity and exercise performance (Fig. 2). This is supported by the finding that there was a positive correlation between LC3-II content and the conversion of glycolytic type IIX muscle fibers toward the more oxidative type IIA fibers in response to endurance exercise training in rats (Tam et al. 2015).

\section{Muscle Mass}

Given the reported effects of resistance exercise on regulation of autophagy, it can be speculated that autophagy-induced amino acid replenishment contributes to the hypertrophic response to resistance exercise training. In addition, the ability of resistance exercise training to preserve muscle mass during aging has been associated with regulation of autophagy (Luo et al. 2013). Thus, aged rats subjected to 9 weeks of weightbearing exercise had higher muscle cross-sec- 
Autophagy-Dependent Beneficial Effects of Exercise

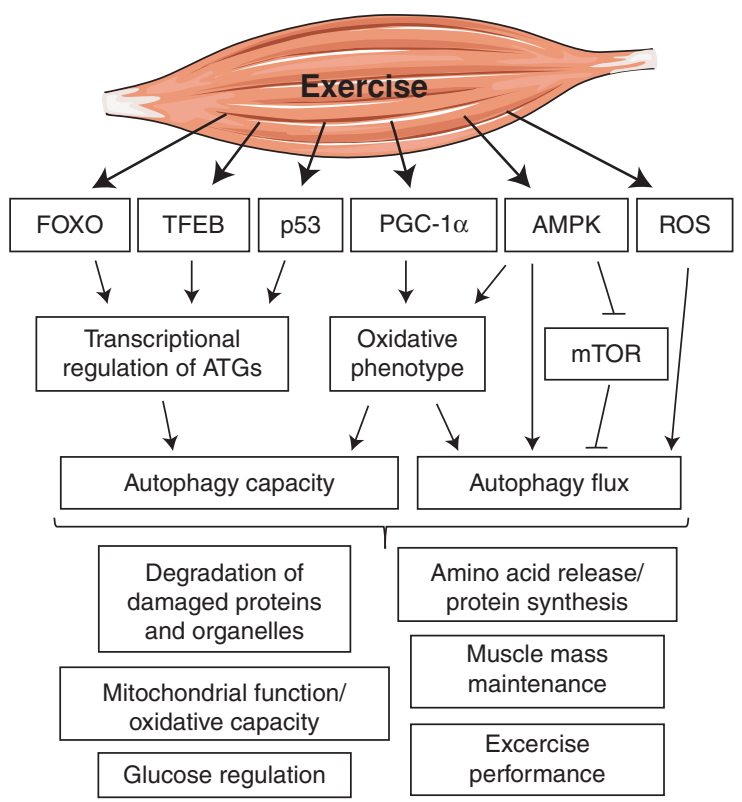

Figure 2. Overview of molecular mediators of exercise-induced autophagy and the autophagy-dependent beneficial effects of exercise. Exercise-induced activation of autophagy in skeletal muscle has been shown to be influenced by transcriptional regulation of autophagy-related genes (ATGs) mediated by forkhead box O (FOXO) family of transcription factors, transcription factor EB (TFEB), p53, and peroxisome proliferatoractivated receptor $\gamma$ coactivator $1 \alpha(\mathrm{PGC}-1 \alpha)$ to increase the autophagic capacity. The oxidative phenotype of skeletal muscle is linked to autophagy capacity/flux and is positively regulated by the exercise responsive $5^{\prime}$ AMP-activated protein kinase (AMPK) and PGC- $1 \alpha$. AMPK and mammalian target of rapamycin (mTOR) act as an activator and suppressor of exercise-induced autophagy, respectively. Exercise-induced generation of reactive oxygen species (ROS) seems to serve as a signal for autophagy activation. Autophagy-dependent beneficial effects of exercise include degradation of oxidatively damaged proteins and organelles, improved mitochondrial oxidative capacity, improved glucose regulation, protein synthesis, preservation of muscle strength and mass, and improved endurance exercise performance.

tional area and strength as well as higher cathepsin L activity, Beclin-1, ATG5 and ATG7 protein content, whereas LC3-II and p62 protein content was lower than in aged untrained rats, altogether indicating increased basal autophagic flux with resistance exercise training (Luo et al. 2013). Furthermore, mice lacking collagen-type VI $\alpha$ (COL6A KO) with impaired autophagy have been shown to display a blunted exerciseinduced increase in LC3II/LC3I with concomitant accumulation of dysfunctional mitochondria, altered mitochondrial network (based on SDH staining), muscle fiber degeneration, and decreased muscle strength (Grumati et al. 2010, 2011). These observations showing that an exercise bout elicited extensive muscle damage in COL6A KO mice, but not WT mice, in- dicate that exercise-induced autophagy is an important myoprotective process. Accordingly, 3 months of exercise training, increasing SDH staining in WT skeletal muscle, reduced skeletal muscle LC3-II/I and exacerbated the dystrophic phenotype with extensive muscle degradation in COL6A KO mice (Grumati et al. 2011). Taken together, this suggests that exercise-induced autophagy is crucial for maintaining muscle mass, ultrastructure, and function (Fig. 2).

\section{Glucose Regulation}

A few studies have examined the role of exercisemediated autophagy on metabolic adjustments in response to exercise. The observation that 
plasma lactate, glucose, and free fatty acid concentrations after exercise were unaffected by inducible muscle-specific KO of ATG7 with impaired exercise-induced autophagy (Lo et al. 2014) suggests that autophagy is not required for exercise-induced adjustments in circulating substrates and metabolites. In contrast, pharmacological inhibition of autophagy reduced insulin-stimulated glucose uptake in $\mathrm{C} 2 \mathrm{C} 12$ myotubes (Liu et al. 2015), supporting a role of autophagy in glucose regulation. In accordance, the impaired exercise-induced activation of autophagy in BCL AAA and ATG6 ${ }^{+/-}$mice was associated with reduced GLUT4 translocation to the sarcolemma in response to exercise and lower radiolabeled glucose uptake in the isolated soleus muscle. This suggests an association between exercise-regulated autophagy and glucose uptake in skeletal muscle. BCL2 AAA mutated mice also showed impaired exercise training-mediated protection against high fat diet-induced glucose intolerance ( $\mathrm{He}$ et al. 2012), further supporting that exercise-induced activation of autophagy is required for the beneficial effects of exercise on glucose metabolism (Fig. 2).

\section{ROS Protection}

The findings that COL6A KO mice had impaired exercise-induced activation of autophagy and accumulated damaged mitochondria (Grumati et al. 2011) suggest that exercise-induced autophagy serves to remove dysfunctional organelles. In accordance, skeletal muscle protein carbonylation (used as marker of oxidative damage) was shown to be reduced in the recovery period from a single exercise bout coinciding with an increase in LC3-II (Halling et al. 2016). This indicates that exercise-induced autophagy plays a role in removing oxidized proteins to prevent the accumulation of dysfunctional proteins. Furthermore, muscle-specific ATG7 KO mice have been shown to exhibit higher ROS accumulation and mitochondrial membrane depolarization in skeletal muscle than WT mice after eccentric contractions (Lo et al. 2014). This supports that the level of reactive oxygen species may play a role in the regu- lation of autophagy (Fig. 2). It should be noted that antioxidant treatment did not prevent exercise-induced activation of mitophagy and the ability of exercise to restore the mitochondrial membrane potential (Lo et al. 2014), showing that additional factors must also be involved. However, $\mathrm{N}$-acetylcysteine (NAC) antioxidant treatment reduced basal mitophagy in mice (Lo et al. 2014; Qi et al. 2014). This may suggest a mechanism explaining the observation that antioxidant supplementation can have adverse effects on exercise performance as well as metabolic adaptations to exercise training (GomezCabrera et al. 2008; Ristow et al. 2009; Gliemann et al. 2013; Olesen et al. 2014).

\section{Disease States}

The impact of exercise-induced autophagy has also been studied through inhibition of autophagy by treatment with the lysosomal inhibitor chloroquine 5 days/week for 16 weeks resulting in sporadic inclusion body myositis, a condition causing muscle weakness and wasting (Kwon et al. 2015). Resistance exercise training was shown to improve muscle strength in chloroquine-treated rats and to prevent chloroquine-induced increases in ATG6 (Beclin-1) and p62 (Kwon et al. 2015). This suggests that resistance exercise training modulates autophagy in atrophying skeletal muscle with potential protective effects on muscle function. Although exercise-induced autophagy normally seems to exert beneficial effects, uncontrolled enhancement of autophagy may lead to muscle wasting and oxidative stress. Hence, treating rats with the autophagy-stimulating antitumor agent doxorubicin was shown to increase LC3-II/I, oxidative stress, and muscle degradation. However, exercise training prevented doxorubicin-induced apoptosis, and elevated the mRNA and protein content of autophagy genes in skeletal muscle (Smuder et al. 2011). This suggests that, in addition to the bona fide autophagy-dependent beneficial effects of exercise, exercise training can contribute in adjusting the level of autophagy in disease states, which may serve to reduce the disease burden. 


\section{CONCLUDING REMARKS}

Numerous studies have provided evidence that autophagy is activated in skeletal muscle in response to exercise. The exercise-induced regulation of autophagy includes both increased autophagy flux as well as transcriptional activation of important autophagy genes potentially resulting in enhanced autophagy capacity. Furthermore, exercise-induced autophagy seems to be involved in mediating many of the beneficial effects of exercise. However, much more remains to be investigated regarding both the molecular mechanisms involved in acute activation of autophagy and the resulting effects. In particular, the potential impact of autophagy on exercise performance and metabolic adaptations in humans is not clear.

\section{REFERENCES}

Bertaggia E, Coletto L, Sandri M. 2012. Posttranslational modifications control FoxO3 activity during denervation. Am J Physiol Cell Physiol 302: C587-C596.

Biolo G, Maggi SP, Williams BD, Tipton LD, Wolfe RR. 1995. Increased rates of muscle protein turnover and amino acid transport after resistance exercise in humans. Am J Physiol 268: E514-E520.

Brunet A, Bonni A, Zigmond MJ, Lin MZ, Juo P, Hu LS, Anderson MJ, Arden KC, Blenis J, Greenberg ME. 1999. Akt promotes cell survival by phosphorylating and inhibiting a Forkhead transcription factor. Cell 96: 857868.

Chesley A, MacDougall JD, Tarnopolsky MA, Atkinson SA, Smith K. 1992. Changes in human muscle protein synthesis after resistance exercise. J Appl Physiol (1985) 73: $1383-1388$.

Dickinson JM, Reidy PT, Gundermann DM, Borack MS, Walker DK, D’Lugos AC, Volpi E, Rasmussen BB. 2016. The impact of post exercise essential amino acid ingestion on the ubiquitin proteasome and autophagosomal-lysosomal systems in skeletal muscle of older men. J Appl Physiol (1985) doi: 10.1152/japplphysiol.00632.2016.

Egan DF, Shackelford DB, Mihaylova MM, Gelino S, Kohnz RA, Mair W, Vasquez DS, Joshi A, Gwinn DM, Taylor R, et al. 2011. Phosphorylation of ULK1 (hATG1) by AMPactivated protein kinase connects energy sensing to mitophagy. Science 331: 456-461.

Francaux M, Demeulder B, Naslain D, Fortin R, Lutz O, Caty G, Deldicque L. 2016. Aging reduces the activation of the mTORC1 pathway after resistance exercise and protein intake in human skeletal muscle: Potential role of REDD1 and impaired anabolic sensitivity. Nutrients. 8: $\mathrm{E} 47$.

Fritzen AM, Madsen AB, Kleinert M, Treebak JT, Lundsgaard AM, Jensen TE, Richter EA, Wojtaszewski J, Kiens B, Frosig C. 2015. Regulation of autophagy in human skeletal muscle-Effects of exercise, exercise training and insulin stimulation. J Physiol 594: 745-761.

Fry CS, Drummond MJ, Glynn EL, Dickinson JM, Gundermann DM, Timmerman KL, Walker DK, Volpi E, Rasmussen BB. 2013. Skeletal muscle autophagy and protein breakdown following resistance exercise are similar in younger and older adults. J Gerontol A Biol Sci Med Sci 68: 599-607.

Gliemann L, Schmidt JF, Olesen J, Bienso RS, Peronard SL, Grandjean SU, Mortensen SP, Nyberg M, Bangsbo J, Pilegaard, et al. 2013. Resveratrol blunts the positive effects of exercise training on cardiovascular health in aged men. J Physiol 591: 5047-5059.

Glynn EL, Fry CS, Drummond MJ, Dreyer HC, Dhanani S, Volpi E, Rasmussen BB. 2010. Muscle protein breakdown has a minor role in the protein anabolic response to essential amino acid and carbohydrate intake following resistance exercise. Am J Physiol Regul Integr Comp Physiol 299: R533-R540.

Gollnick PD, Armstrong RB, Saubert CW, Piehl K, Saltin B. 1972. Enzyme activity and fiber composition in skeletal muscle of untrained and trained men. J Appl Physiol 33: 312-319.

Gomez-Cabrera MC, Domenech E, Romagnoli M, Arduini A, Borras C, Pallardo FV, Sastre J, Vina J. 2008. Oral administration of vitamin $\mathrm{C}$ decreases muscle mitochondrial biogenesis and hampers training-induced adaptations in endurance performance. Am J Clin Nutr 87: $142-149$.

Grumati P, Coletto L, Sabatelli P, Cescon M, Angelin A, Bertaggia E, Blaauw B, Urciuolo A, Tiepolo T, Merlini $\mathrm{L}$, et al. 2010. Autophagy is defective in collagen VI muscular dystrophies, and its reactivation rescues myofiber degeneration. Nat Med 16: 1313-1320.

Grumati P, Coletto L, Schiavinato A, Castagnaro S, Bertaggia E, Sandri M, Bonaldo P. 2011. Physical exercise stimulates autophagy in normal skeletal muscles but is detrimental for collagen VI-deficient muscles. Autophagy 7: 14151423.

Halling JF, Ringholm S, Nielsen MM, Overby P, Pilegaard H. 2016. PGC-1 $\alpha$ promotes exercise-induced autophagy in mouse skeletal muscle. Physiol Rep 4: e12698.

He C, Bassik MC, Moresi V, Sun K, Wei Y, Zou Z, An Z, Loh J, Fisher J, Sun Q, et al. 2012. Exercise-induced BCL2-regulated autophagy is required for muscle glucose homeostasis. Nature 481: 511-515.

Henriksson J, Reitman JS. 1977. Time course of changes in human skeletal muscle succinate dehydrogenase and cytochrome oxidase activities and maximal oxygen uptake with physical activity and inactivity. Acta Physiol Scand 99: 91-97.

Holloszy JO. 1967. Biochemical adaptations in muscle. Effects of exercise on mitochondrial oxygen uptake and respiratory enzyme activity in skeletal muscle. $J$ Biol Chem 242: 2278-2282.

Jamart C, Benoit N, Raymackers JM, Kim HJ, Kim CK, Francaux M. 2012a. Autophagy-related and autophagyregulatory genes are induced in human muscle after ultraendurance exercise. Eur J Appl Physiol 112: 31733177.

Jamart C, Francaux M, Millet GY, Deldicque L, Frere D, Feasson L. 2012b. Modulation of autophagy and ubiqui- 
tin-proteasome pathways during ultra-endurance running. J Appl Physiol (1985) 112: 1529-1537.

Jamart C, Naslain D, Gilson H, Francaux M. 2013. Higher activation of autophagy in skeletal muscle of mice during endurance exercise in the fasted state. Am J Physiol Endocrinol Metab 305: E964-E974.

Kenzelmann BD, Spano MS, Bieging KT, Jiang D, Dusek RL, Brady CA, Sidow A, Attardi LD. 2013. Global genomic profiling reveals an extensive p53-regulated autophagy program contributing to key p53 responses. Genes Dev 27: 1016-1031.

Kim J, Kundu M, Viollet B, Guan KL. 2011. AMPK and mTOR regulate autophagy through direct phosphorylation of Ulk1. Nat Cell Biol 13: 132-141.

Klionsky DJ, Abdelmohsen K, Abe A, Abedin MJ, Abeliovich $\mathrm{H}$, Acevedo AA, Adachi H, Adams CM, Adams PD, Adel $\mathrm{K}$, et al. 2016. Guidelines for the use and interpretation of assays for monitoring autophagy. Autophagy 12: 1-222.

Ktistakis NT, Tooze SA. 2016. Digesting the expanding mechanisms of autophagy. Trends Cell Biol 26: 624-635.

Kwon I, Lee Y, Cosio-Lima LM, Cho JY, Yeom DC. 2015. Effects of long-term resistance exercise training on autophagy in rat skeletal muscle of chloroquine-induced sporadic inclusion body myositis. J Exerc Nutrition Biochem 19: 225-234.

Lin J, Handschin C, Spiegelman BM. 2005. Metabolic control through the PGC-1 family of transcription coactivators. Cell Metab 1: 361-370.

Lira VA, Okutsu M, Zhang M, Greene NP, Laker RC, Breen DS, Hoehn KL, Yan Z. 2013. Autophagy is required for exercise training-induced skeletal muscle adaptation and improvement of physical performance. FASEB $J$ 27: 4184-4193.

Liu X, Niu Y, Yuan H, Huang J, Fu L. 2015. AMPK binds to Sestrins and mediates the effect of exercise to increase insulin-sensitivity through autophagy. Metabolism 64: 658-665.

Lo VF, Carnio S, Vainshtein A, Sandri M. 2014. Autophagy is not required to sustain exercise and PRKAA1/AMPK activity but is important to prevent mitochondrial damage during physical activity. Autophagy 10: 1883-1894.

Louis E, Raue U, Yang Y, Jemiolo B, Trappe S. 2007. Time course of proteolytic, cytokine, and myostatin gene expression after acute exercise in human skeletal muscle. J Appl Physiol (1985) 103: 1744-1751.

Luo L, Lu AM, Wang Y, Hong A, Chen Y, Hu J, Li X, Qin ZH. 2013. Chronic resistance training activates autophagy and reduces apoptosis of muscle cells by modulating IGF-1 and its receptors, Akt/mTOR and Akt/FOXO3a signaling in aged rats. Exp Gerontol 48: 427-436.

MacKenzie MG, Hamilton DL, Murray JT, Taylor PM, Baar K. 2009. mVps34 is activated following high-resistance contractions. J Physiol 587: 253-260.

Maiuri MC, Malik SA, Morselli E, Kepp O, Criollo A, Mouchel PL, Carnuccio R, Kroemer G. 2009. Stimulation of autophagy by the p53 target gene Sestrin2. Cell Cycle 8: 1571-1576.

Mammucari C, Milan G, Romanello V, Masiero E, Rudolf R, Del PP, Burden SJ, Di LR, Sandri C, Zhao J, et al. 2007. FoxO3 controls autophagy in skeletal muscle in vivo. Cell Metab 6: 458-471.
Masschelein E, Van TR, D’Hulst G, Hespel P, Thomis M, Deldicque L. 2014. Acute environmental hypoxia induces LC3 lipidation in a genotype-dependent manner. FASEB J 28: $1022-1034$.

Medina DL, Di Paola S, Peluso I, Armani A, De Stefani D, Venditti R, Montefusco S, Scotto-Rosato A, Prezioso C, Forrester A, et al. 2015. Lysosomal calcium signalling regulates autophagy through calcineurin and TFEB. Nat Cell Biol 17: 288-299.

Meley D, Bauvy C, Houben-Weerts JH, Dubbelhuis PF, Helmond MT, Codogno P, Meijer. 2006. AMP-activated protein kinase and the regulation of autophagic proteolysis. J Biol Chem 281: 34870-34879.

Mizushima N, Komatsu M. 2011. Autophagy: Renovation of cells and tissues. Cell 147: 728-741.

Mizushima N, Yoshimori T. 2007. How to interpret LC3 immunoblotting. Autophagy 3: 542-545.

Moller AB, Vendelbo MH, Christensen B, Clasen BF, Bak AM, Jorgensen JO, Moller N, Jessen N. 2015. Physical exercise increases autophagic signaling through ULK1 in human skeletal muscle. J Appl Physiol (1985) 118: 971-979.

Ogasawara R, Fujita S, Hornberger TA, Kitaoka Y, Makanae Y, Nakazato K, Naokata I. 2016. The role of mTOR signalling in the regulation of skeletal muscle mass in a rodent model of resistance exercise. Sci Rep 6: 31142 .

Ogborn DI, McKay BR, Crane JD, Safdar A, Akhtar M, Parise G, Tarnopolsky MA. 2015. Effects of age and unaccustomed resistance exercise on mitochondrial transcript and protein abundance in skeletal muscle of men. Am J Physiol Regul Integr Comp Physiol 308: R734-R741.

Olesen J, Gliemann L, Bienso R, Schmidt J, Hellsten Y, Pilegaard H. 2014. Exercise training, but not resveratrol, improves metabolic and inflammatory status in skeletal muscle of aged men. J Physiol 592: 1873-1886.

Pagano AF, Py G, Bernardi H, Candau RB, Sanchez AM. 2014. Autophagy and protein turnover signaling in slow-twitch muscle during exercise. Med Sci Sports Exerc 46: $1314-1325$.

Phillips SM, Tipton KD, Aarsland A, Wolf SE, Wolfe RR. 1997. Mixed muscle protein synthesis and breakdown after resistance exercise in humans. Am J Physiol 273: E99-E107.

Pietrocola F, Izzo V, Niso-Santano M, Vacchelli E, Galluzzi L, Maiuri MC, Kroemer G. 2013. Regulation of autophagy by stress-responsive transcription factors. Semin Cancer Biol 23: 310-322.

Pilegaard H, Saltin B, Neufer PD. 2003. Exercise induces transient transcriptional activation of the PGC- $1 \alpha$ gene in human skeletal muscle. J Physiol 546: 851-858.

Qi Z, He Q, Ji L, Ding S. 2014. Antioxidant supplement inhibits skeletal muscle constitutive autophagy rather than fasting-induced autophagy in mice. Oxid Med Cell Longev 2014: 315896.

Reynolds TH, Merrell E, Cinquino N, Gaugler M, Ng L. 2012. Disassociation of insulin action and Akt/FOXO signaling in skeletal muscle of older Akt-deficient mice. Am J Physiol Regul Integr Comp Physiol 303: R1186R1194.

Ristow M, Zarse K, Oberbach A, Kloting N, Birringer M, Kiehntopf M, Stumvoll M, Kahn CR, Bluher M. 2009. 
Antioxidants prevent health-promoting effects of physical exercise in humans. Proc Natl Acad Sci 106: 86658670.

Rubinsztein DC, Cuervo AM, Ravikumar B, Sarkar S, Korolchuk V, Kaushik S, Klionsky DJ. 2009. In search of an "autophagomometer." Autophagy 5: 585-589.

Saleem A, Hood DA. 2013. Acute exercise induces tumour suppressor protein $\mathrm{p} 53$ translocation to the mitochondria and promotes a p53-Tfam-mitochondrial DNA complex in skeletal muscle. J Physiol 591: 3625-3636.

Saleem A, Adhihetty PJ, Hood DA. 2009. Role of p53 in mitochondrial biogenesis and apoptosis in skeletal muscle. Physiol Genomics 37: 58-66.

Saleem A, Carter HN, Hood DA. 2014. p53 is necessary for the adaptive changes in cellular milieu subsequent to an acute bout of endurance exercise. Am J Physiol Cell Physiol 306: C241-C249.

Salminen A, Vihko V. 1984. Autophagic response to strenuous exercise in mouse skeletal muscle fibers. Virchows Arch B Cell Pathol Incl Mol Pathol 45: 97-106.

Sanchez AM, Csibi A, Raibon A, Cornille K, Gay S, Bernardi H, Candau R. 2012. AMPK promotes skeletal muscle autophagy through activation of forkhead FoxO3a and interaction with Ulk1. J Cell Biochem 113: 695-710.

Schwalm C, Jamart C, Benoit N, Naslain D, Premont C Prevet J, Van TR, Deldicque L, Francaux M. 2015. Activation of autophagy in human skeletal muscle is dependent on exercise intensity and AMPK activation. FASEB 29: 3515-3526.

Settembre C, Di Malta C, Polito VA, Garcia Arencibia M, Vetrini F, Erdin S, Erdin SU, Huynh T, Medina D, Colella P, et al. 2011. TFEB links autophagy to lysosomal biogenesis. Science 332: 1429-1433.

Smiles WJ, Areta JL, Coffey VG, Phillips SM, Moore DR, Stellingwerff T, Burke LM, Hawley JA, Camera DM. 2015 Modulation of autophagy signaling with resistance exercise and protein ingestion following short-term energy deficit. Am J Physiol Regul Integr Comp Physiol 309: R603-R612.

Smiles WJ, Parr EB, Coffey VG, Lacham-Kaplan O, Hawley JA, Camera DM. 2016. Protein coingestion with alcohol following strenuous exercise attenuates alcohol-induced intramyocellular apoptosis and inhibition of autophagy. Am J Physiol Endocrinol Metab doi: 10.1152/ajpendo. 00303.2016.
Autophagy-Dependent Beneficial Effects of Exercise

Smuder AJ, Kavazis AN, Min K, Powers SK. 2011. Exercise protects against doxorubicin-induced markers of autophagy signaling in skeletal muscle. J Appl Physiol (1985) 111: 1190-1198.

Tachtsis B, Smiles WJ, Lane SC, Hawley JA, Camera DM. 2016. Acute endurance exercise induces nuclear p53 abundance in human skeletal muscle. Front Physiol 7: 144.

Tam BT, Pei XM, Yu AP, Sin TK, Leung KK, Au KK, Chong JT, Yung BY, Yip SP, Chan LW, et al. 2015. Autophagic adaptation is associated with exercise-induced fibre-type shifting in skeletal muscle. Acta Physiol (Oxf) 214: $221-$ 236.

Tasdemir E, Chiara MM, Morselli E, Criollo A, D'Amelio M, Djavaheri-Mergny M, Cecconi F, Tavernarakis N, Kroemer G. 2008. A dual role of p53 in the control of autophagy. Autophagy 4: 810-814.

Ulbricht A, Gehlert S, Leciejewski B, Schiffer T, Bloch W, Hohfeld J. 2015. Induction and adaptation of chaperoneassisted selective autophagy CASA in response to resistance exercise in human skeletal muscle. Autophagy 11: $538-546$.

Vainshtein A, Desjardins EM, Armani A, Sandri M, Hood DA. 2015a. PGC-1 $\alpha$ modulates denervation-induced mitophagy in skeletal muscle. Skelet Muscle 5: 9.

Vainshtein A, Tryon LD, Pauly M, Hood DA. 2015b. Role of PGC-1 $\alpha$ during acute exercise-induced autophagy and mitophagy in skeletal muscle. Am J Physiol Cell Physiol 308: C710-C719.

Wang P, Li CG, Qi Z, Cui D, Ding S. 2015. Acute exercise induced mitochondrial $\mathrm{H}_{2} \mathrm{O}_{2}$ production in mouse skeletal muscle: Association with $\mathrm{p}^{66 \mathrm{Shc}}$ and FOXO3a signaling and antioxidant enzymes. Oxid Med Cell Longev 2015: 536456.

Wu Z, Puigserver P, Andersson U, Zhang C, Adelmant G, Mootha V, Troy A, Cinti S, Lowell B, Scarpulla RC, et al. 1999. Mechanisms controlling mitochondrial biogenesis and respiration through the thermogenic coactivator PGC-1. Cell 98: 115-124.

Youle RJ, Narendra DP. 2011. Mechanisms of mitophagy. Nat Rev Mol Cell Biol 12: 9-14.

Zhao M, Klionsky DJ. 2011. AMPK-dependent phosphorylation of ULK1 induces autophagy. Cell Metab 13: 119120. 


\section{$\&_{\mathrm{CSH}}^{\infty} \&$ Cold Spring Harbor

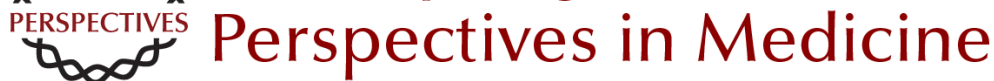

\section{Autophagy-Dependent Beneficial Effects of Exercise}

Jens Frey Halling and Henriette Pilegaard

Cold Spring Harb Perspect Med 2017; doi: 10.1101/cshperspect.a029777 originally published online March 7, 2017

\section{Subject Collection The Biology of Exercise}

Exosomes as Mediators of the Systemic

Adaptations to Endurance Exercise Adeel Safdar and Mark A. Tarnopolsky

Molecular Basis of Exercise-Induced Skeletal

Muscle Mitochondrial Biogenesis: Historical

Advances, Current Knowledge, and Future

Challenges

Christopher G. R. Perry and John A. Hawley

Exercise Metabolism: Fuels for the Fire Mark Hargreaves and Lawrence L. Spriet

Health Benefits of Exercise Gregory N. Ruegsegger and Frank W. Booth

Molecular Regulation of Exercise-Induced Muscle

Fiber Hypertrophy

Marcas M. Bamman, Brandon M. Roberts and Gregory R. Adams

Physiological Redundancy and the Integrative

Responses to Exercise Michael J. Joyner and Jerome A. Dempsey

On the Run for Hippocampal Plasticity C'iana Cooper, Hyo Youl Moon and Henriette van Praag

Molecular Basis for Exercise-Induced Fatigue: The Importance of Strictly Controlled Cellular $\mathrm{Ca}$

2+ Handling

Arthur J. Cheng, Nicolas Place and Håkan

Westerblad
Effects of Exercise and Aging on Skeletal Muscle Giovanna Distefano and Bret $H$. Goodpaster

Muscle-Adipose Tissue Cross Talk

Kristin I. Stanford and Laurie J. Goodyear

Performance Fatigability: Mechanisms and Task Specificity

Sandra K. Hunter

Adaptations to Endurance and Strength Training David C. Hughes, Stian Ellefsen and Keith Baar

The Bioenergetics of Exercise

$P$. Darrell Neufer

Effects of Exercise on Vascular Function,

Structure, and Health in Humans

Daniel J. Green and Kurt J. Smith

Control of Muscle Metabolism by the Mediator

Complex

Leonela Amoasii, Eric N. Olson and Rhonda

Bassel-Duby

Theoretical and Biological Evaluation of the Link between Low Exercise Capacity and Disease Risk Lauren Gerard Koch and Steven L. Britton

For additional articles in this collection, see http://perspectivesinmedicine.cshlp.org/cgi/collection/ 